$$
\text { "gjone" — 2004/7/22 — 15:27 — page } 117 \text { — \#1 }
$$

\title{
Process or object? Ways of solving mathematical problems using CAS
}

\author{
Gunnar GJone
}

\begin{abstract}
Graphing and symbol manipulating calculators are now a part of mathematics education in many countries. In Norway symbol manipulating calculators have been used at various exams in upper secondary education. An important finding in mathematics education is the duality of mathematical entities - processes and objects. Building on the theoretical development by Anna Sfard and others, the students' solutions on exam problems in upper secondary education are discussed with reference to procedural and structural knowledge.
\end{abstract}

Key words and phrases: procedural/conceptual knowledge, computer algebra systems, calculators.

ZDM Subject Classification: D60, U70.

\section{Introduction}

In his lecture at the International Congress of Mathematicians (ICM) in Berlin in 1998, Mogens Niss was presenting for mathematicians what he considered was important findings in mathematics education. One of these important findings was the object - process duality found in mathematics and the problems encountered by students facing transition from process to object. This topic has been the subject of discussion and development and has been given several formulations. Mathematical entities can be considered as processes or objects, and the relationship between them has been the subject of several studies and various relations have been postulated. Several authors have argued that process (procedural knowledge) is necessary for building concepts (conceptual knowledge).

Copyright (c) 2004 by University of Debrecen
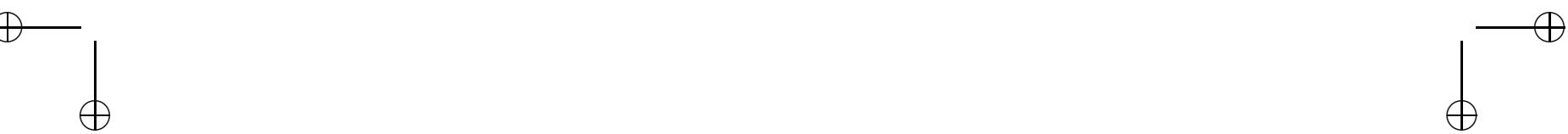


$$
\text { "gjone" — 2004/7/22 — 15:27 — page } 118 \text { — \#2 }
$$

Graphing calculators and computer algebra systems (CAS) are relatively new to mathematics education. Even if the use of computers in mathematics has been developed over the last 50-60 years, it is only since the 1970s that technology has played an increasingly important part in mathematics education. In the history of technology in mathematics education, the year 1988 is an important year. The computer programs Mathematica and Cabri Geomètre were released, and computer algebra systems and geometry tools became available on personal computers.

Linked to the process - object duality are also different forms of knowledge: procedural knowledge, structural knowledge (sometimes also denoted conceptual.knowledge).

We observe that it was about the same time that papers about the object - process duality appeared in various publications, although the topic had also been discussed earlier.

\section{The process - object duality}

\section{Forms of knowledge}

There is a large amount of literature on this topic, and different authors have used slightly different terms, and also with some variation of meaning. However, it seems to be a common "core". The following definition of procedural is given in Gray \& Tall [1]:

Procedural aspects of mathematics focus on routine manipulation of objects that are represented either by concrete materials, spoken words, written symbols, or mental images. It is relatively easy to see if such procedures are carried out adequately, and performance in similar tasks is often taken as a measure of attainment in these skills.

They further refer to the definition given by Hiebert and Lefevre [2] for conceptual knowledge:

a connected web ... a network in which the linking relationships are as prominent as the discrete pieces of information ... . A unit of conceptual knowledge cannot be an isolated piece of information; by definition it is part of conceptual knowledge only if the holder recognizes its relationship to other pieces of information (pp. 3-4).

Anna Sfard is perhaps the most well known contributor to this distinction. She argues that abstract mathematical notions can be conceived in two different ways, 


$$
\text { "gjone" — 2004/7/22 — 15:27 — page } 119 \text { — \#3 }
$$

structurally as objects or operationally as processes. She is also concerned about the sequence in which these forms of knowledge occur. In her view for most people the operational comes before the structural, and the transition from a "process" conception to an "object" conception is difficult.

Seeing a mathematical entity as an object means being capable of referring to it as if it was a real thing - a static structure, existing somewhere in space and time. It also means being able to recognize the idea "at a glance" and to manipulate it as a whole, without going into details (Sfard, [5], p. 4).

In several papers Sfard has written about the operational - structural duality of mathematical conceptions, e.g. Sfard [4]-[6]). Looking at the historical development of mathematical concepts she introduces the notion of reification:

... the idea of turning a process into an autonomous entity should emerge, and finally to see this new entity as an integrated, object-like whole must be acquired (Sfard, [5], p. 18).

Eddie M. Gray and David O. Tall also discuss this dichotomy in their well known paper from 1994 (Gray \& Tall, [1]). They use the term encapsulation to denote the same phenomena, but introduce an "extended" terminology. Referring to mathematicians who "employ the simple device of using the same notation to represent both a process and the product of that process" (p. 119), they introduce the notion procept:

We propose the following preliminary definition: An elementary procept is the amalgam of three components: a process that produces a mathematical object, and a symbol that represents either the process or the object (p. 121).

They propose a somewhat extended model differentiating between less and more able students. They introduce the notion proceptual divide:

The less able child who is fixed in process can only solve problems at the next level up by coordinating sequential processes.

.

The more able, proceptual thinker is faced with an easier task. The symbols for sum and product again represent numbers. Thus counting, addition, and multiplication are operating on the same procept, which can be decomposed into process for calculation purposes whenever desired (p. 135).

In his presentation at ICME 8 in Seville in 1996 David Tall elaborated the notion.
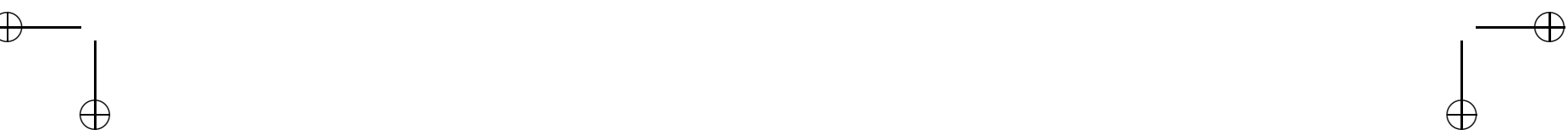


$$
\text { "gjone" — 2004/7/22 — 15:27 — page } 120 \text { — \#4 }
$$

Procepts allow the individual not only to carry out procedures, but to regard symbols as mental objects, so they can not only do mathematics, They can also think about the concepts (Tall, [7], p. 77).

We will return to the notion of precept in the discussion at the end of the article. Sfard states that reification is difficult to attain, but it is also needed to attain relational understanding (Sfard, [6], p. 4). Concerning this process there are different views of the relationship between procedural and structural knowledge. Several researchers will argue that procedural knowledge is necessary for structural knowledge, e.g. in Hiebert [2]), Gray \& Tall [1]. This is also a view that Anna Sfard advocates. What are then the implications for teaching derived from this distinction?

In (Sfard, [6]) we find two principles for teaching:

(1) New concepts should not be introduced by help of their structural description

(2) Structural conception should not be required as long as the student can do without it (Sfard, [6], p. 6).

Based on the second principle we can ask the question if students with a graphing calculator can do without a structural conception. Where one earlier relied on seeing mathematical entities as objects, the graphing calculator - for these students and for this problem - may have eliminated the need for a structural conception of a function.

\section{Problem formulation}

The graphing calculator has made an impact in upper secondary mathematics education in many countries. The most recent development, a graphing calculator with CAS or symbol manipulating calculator (abbreviated SMP in this paper) is starting to make impact as well.

Many mathematical objects - like a function graph - is usually in mathematics education introduced as a process, but on a SMC or graphing calculator a graph is presented as an object directly with no mathematical process leading to it. The graph of a function is presented directly from the algebraic expression. Anna Sfard is also discussing this in her [5] paper:

The computer program seems to correspond to an operational conception rather than a structural, since it presents the function as a computational process, not as a unified entity. In the graphic representation, on the other hand, the infinitely many components of the function are combined into a smooth line, so they can be grasped simultaneously as an integrated 


$$
\text { "gjone" — 2004/7/22 — 15:27 — page } 121 \text { — \#5 }
$$

whole: the graph therefore encourages a structural approach (Sfard, [5], p. 6).

The symbol manipulating calculator (SMC) in particular is a powerful tool in making many procedural aspects "concrete".

The basic question for this study is how the symbol manipulating calculator influences the reification process. More specifically: What kind of influence does the use of SMC have on students' forms of knowledge in a problem solving situation?

\section{The study}

\section{Background}

The 3 year upper secondary school in Norway has several streams. The general or academic stream has mathematics courses in all three years, but only the first year is compulsory. In each stream there are moreover 2 (or 3) levels of mathematics. There is a basic course in mathematics in the first year. This is the basic course for all upper secondary school students - also for the vocational streams. For the academic streams it functions as the introduction, and it is followed by two possibilities: One course for those who want to continue with mathematics or one course for those who want to end their mathematics course after the first year or have a shorter mathematics course in the second year.

In the school year 1998-1999 the National Examination Board in Norway initiated a study with symbol-manipulating calculators (SMC) in upper secondary mathematics. The main purpose was to investigate the conditions concerning the use of this type of calculators for final exams in mathematics in the second year of upper secondary school. The project has continued since then, involving all three years in upper secondary school.

\section{The Projects}

The rationale for the projects was given by the school authorities:

The purpose with this experiment is to find out if it is possible to construct examination problems and organize test situations that mirrors real challenges and possibilities, and at the same time provide a basis for giving individual marks. (From an "unofficial" letter to teachers in the project, translated by the author.) 


$$
\text { "gjone" — 2004/7/22 — 15:27 — page } 122 \text { — \#6 }
$$

Schools (teachers) could register to participate. For the study the schools themselves had to get or borrow the calculators from companies producing or importing the SMCs. The National Examination Board had some limited funds available for supporting the teachers, and had more of a coordinating function. The projects have been part of a larger study on the use of information technology in different school subjects (e.g. Norwegian, English).

In this project the teachers had volunteered to participate - hence there were teachers interested in technology participating. In this article I will present some result from the following exam:

\begin{tabular}{|l|c|l|}
\hline Course & Year & Equipment \\
\hline $\begin{array}{l}\text { 2nd year upper secondary } \\
\text { mathematics for specialist }\end{array}$ & 1999 & Graphic calculators TI 89 \& TI 92 \\
\hline
\end{tabular}

\section{The exam}

For the students this was a high stake exam, having consequences for their further programs in mathematics, but also in some cases consequences for tertiary education.

Much of the projects were focused on problem construction and student performance on the exam. This type of exam in Norway is usually a 5 hour written exam. For upper secondary mathematics $\left(2^{\text {nd }}\right.$ and $3^{\text {rd }}$ year) all students have graphic calculators as their normal equipment. The students in the projects had the regular exam in mathematics, except that some of the problems were replaced by problems especially designed for the use of SMC (CAS).

The following instructions are stated on the second page of the exam. They were the same for both the regular and the special students using SMC.

\section{Graphs - use of graphing calculator}

State which calculator functions you have used. It is not necessary to give detailed use of keys.

Remember to write the scale and units on the axes if you draw graphs as part of your answers. You do not need to include a table of the values you have calculated for the function unless specially asked to do so.

If you use the calculator to construct graphs, it is sufficient to sketch the shape of the curve in your answer. The sketch must show clearly how you arrived at the answer. 


$$
\text { "gjone" — 2004/7/22 — 15:27 — page } 123 \text { — \#7 }
$$

Process or object? Ways of solving mathematical problems using CAS

(Instructions given to the candidates on the exams, translated by the author)

\section{Method}

The basic method of the study is qualitative. Access has been given to students' exam papers. I was also one of the graders of this exam, working in a group with two other graders. The overall grade given is not important in this study, but discussing with the other graders (of the same exam papers) gave added insight into the students' solutions.

The data - the problems and students' solutions

We will restrict ourselves to three problems.

The main data for the studies have been the students' exam papers. The graders had not access to the name or sex of the students at the time of the grading. Analyzing differences relating to these factors has not been performed for this article.

In the study a questionnaire for teachers was also provided. The answers gave us information on how the teachers reacted. The teachers were in general positive (which they had been from the start). We asked specifically about problem types suitable for these calculators.

Going through the exam papers of the students, we found several interesting relations on how the students used their calculators. Some of these findings relate to the SMCs especially, whereas others relate to graphing calculators in general. By grading the exams we found that actually the regular problems (for the use of ordinary graphic calculators) provided the most interesting material. The special problem for SMCs did not give as many interesting results. Moreover, the special problems were not adequate for differentiating among students.

Depth of water

We studied the solution of the following problem:

\section{Problem 1}

d) The depth of water in a harbour varies with the tide. In a certain 24-hour period the depth is given by the function $f$ where

$$
f(x)=8.50+0.70 \cdot \cos \frac{4 \pi}{25} x
$$




$$
\text { "gjone" — 2004/7/22 — 15:27 — page } 124 \text { — \#8 }
$$

where $x$ is measured in hours and $f(x)$ is measured in meters.

1) What is the difference in depth between high and low tide?

2) How long time is it from one high tide to the next?

(Problem 1d) from $2^{\text {nd }}$ year mathematics exam, spring 1999. Translated by the author.)

Solution of this problem is straightforward without using a graphic calculator (or SMC). The answer to the first question could easily be obtained by noting that the cosine function varies between -1 and +1 , hence the answer is $0.70 \mathrm{~m}+0.70 \mathrm{~m}=$ $1.40 \mathrm{~m}$. The answer to the second problem is found by similarly noting that the cosine function has a maximum value for $x=0$ and a period of $2 \pi$, hence the next value for a maximum is $\frac{4 \pi}{25} x=2 \pi$ or $x=12.5$.

What the majority of the students did was to graph the function, then using either the Trace function or MAX/Min of functions to find the difference. To present the correct answer to this problem the students would draw or sketch the graph indicating which calculator functions they had used. A quite typical answer is presented below.

In one exam paper the following graph was drawn:

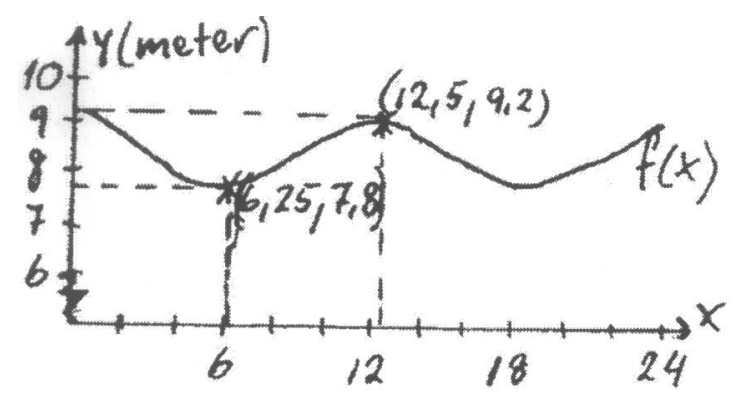

The student wrote the following comment to the graph, which is sketched in the exam paper:

To find how much more shallow the harbour is at low tide than at high tide we subtract the $y$-value in the lowest point (water level by low tide)

from the $y$-value at the top point (high tide).

The difference is $9.2-7.8=1.4$.

Before the presentation of the graph he had given very detailed description on how he had used the calculator to obtain the graph. This was quite common for the students' answers, and the reason was quite clearly the section Graphs - use of graphing calculator, cited above. 


$$
\text { "gjone" — 2004/7/22 — 15:27 — page } 125 \text { — \#9 }
$$

Several of the solutions contained very "elaborate" graphs similar to the one above. For some, writing up the solution to the problem took more than a full page of written text. Many also included quite extensive description on how they used the TRACE-function or the MAX/Min - functions on the calculator. Since this was one of the first problems on the test, it was meant to be an easy task and most students in our sample presented a correct solution. The graders would not differentiate between different types of correct solutions.

A trigonometric identity

\section{Problem 4}

In the triangle $A B C$ we have $\angle A=x, \angle B=2 x$ and $A B=5$.

a) Sketch the triangle $A B C$ for different values of $x$. Explain why $x \in\langle 0, \pi / 3\rangle$

b) Use $\sin (2 x+x)=\sin (3 x)$ to show that $\sin (3 x)=3 \sin x-4 \sin ^{3} x$

(Problem 4 from $2^{\text {nd }}$ year mathematics exam, spring 1999. Translated by the author.)

We will here especially consider problem b). The straightforward "traditional" solution of the problem would be something like the following:

$$
\begin{aligned}
\sin 3 x= & \sin (2 x+x) \\
= & {[\text { then using the formula for } \sin \text { to a sum, }} \\
& \text { and then simplifying to obtain the desired result }]
\end{aligned}
$$

A typical answer that was found was that the students used the calculator to evaluate the equality directly. We give here some of the students' answers (in translation).

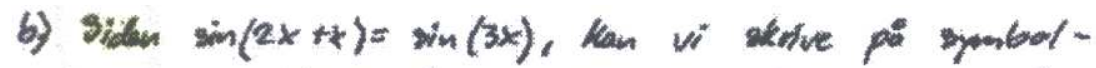 regneren: tCalleat $\left.(\sin (2 x+x))=\frac{6}{1)} \int_{3}\right)$ flect $(3 * \sin (x)-4 *(\sin (x$ $\Rightarrow$ truc. Datte ofl oi at attryalleve or thee for alle vendier ar $x$.}

Author's translation:

Since $\sin 3 x=\sin (2 x+x)$, we can write on our calculator

$t$ Collect $(\sin (2 x+x))=t \operatorname{Collect}\left(3^{*} \sin (x)-4^{*}(\sin (x))^{\wedge} 3\right) \Rightarrow$ true.

This means that the expressions are equal for all values of $x$. 


$$
\text { "gjone" — 2004/7/22 — 15:27 — page } 126 \text { — \#10 }
$$

A similar but "simpler" (?) way of writing the solution was found in some students' "solutions":

I use TI-89 and write

$$
(\sin (3 x)=\sin (2 x+x))=\left(\sin (3 x)=\left(3^{*} \sin (x)-4^{*}(\sin (x))^{\wedge} 3\right)\right)
$$

Pressing ENTER I get true. This shows that the expression in the first

large parenthesis is equal to the expression in the second large parenthesis.

TI-89 simplifies the expression to $\left(\sin (3 x)=\left(3^{*} \sin (x)-4^{*}(\sin (x))^{\wedge} 3\right)\right)$

Some of the students used the traditional approach, but a number of students were attempting a solution similar to the ones presented above. For this problem it was quite clear that the committee making up this exam problem had not taken into account the possibilities of SMCs.

Another type of solution that was found used the graphic capabilities of the calculator. The students were drawing two graphs: One graph of the function $\sin 3 x$ or of the function $\sin (2 x+x)$ and then another graph of $3 \sin x-4 \sin 3 x$. Observing that the graphs coincided they concluded that the functions were equal.

The third problem that we looked into is the last part of problem 4.

\section{Finding a limit}

The concept of limit is important in calculus. The version of the computer program Derive on the TI- 89 and TI-92 is able to compute values of limits.

\section{Problem 4}

d) Show that the area of the triangle can be written as

$$
f(x)=\frac{12.5 \sin (2 x)}{3-4 \sin ^{2} x} \quad x \in\left\langle 0, \frac{\pi}{3}\right\rangle
$$

e) Investigate if the area $F$ has a maximum value. What happens with the area when

$$
x \rightarrow \frac{\pi}{3}
$$

(Problems 4d) \& 4e) from $2^{\text {nd }}$ year mathematics exam, spring 1999. The text translated by the author.)

We will concentrate our discussion on problem 4e). The function in d) is given so that students can answer question e) without having arrived at the correct expression in d). We first note that the solution is straightforward knowing something about the properties of the sin function. As $x$ approaches $\frac{\pi}{3}$ the denominator approaches zero, whereas the numerator approaches a number different from zero. 


$$
\text { "gjone" — 2004/7/22 — 15:27 — page } 127 \text { — \#11 }
$$

Several students observed this fact. We also observe that the result can be obtained from a geometrical reasoning about the triangle $(A B C)$. The definition of the triangle is given above in the second example (A trigonometric identity). However, a number of students entered the expression directly, knowing that the SMC was able to calculate limits.

The most common answer was that the limit was undefined. Several would get the answer "undef(ined)" on their SMC, and then just state that the answer: undefined. One conclusion would be that they were very limited by the SMC, and not trying to reason without it.

We also found several other answers, probably as a consequence that they first got the answer undefined, and were not satisfied with what the SMC presented. Some students remarked this fact in their paper.

One student wrote:

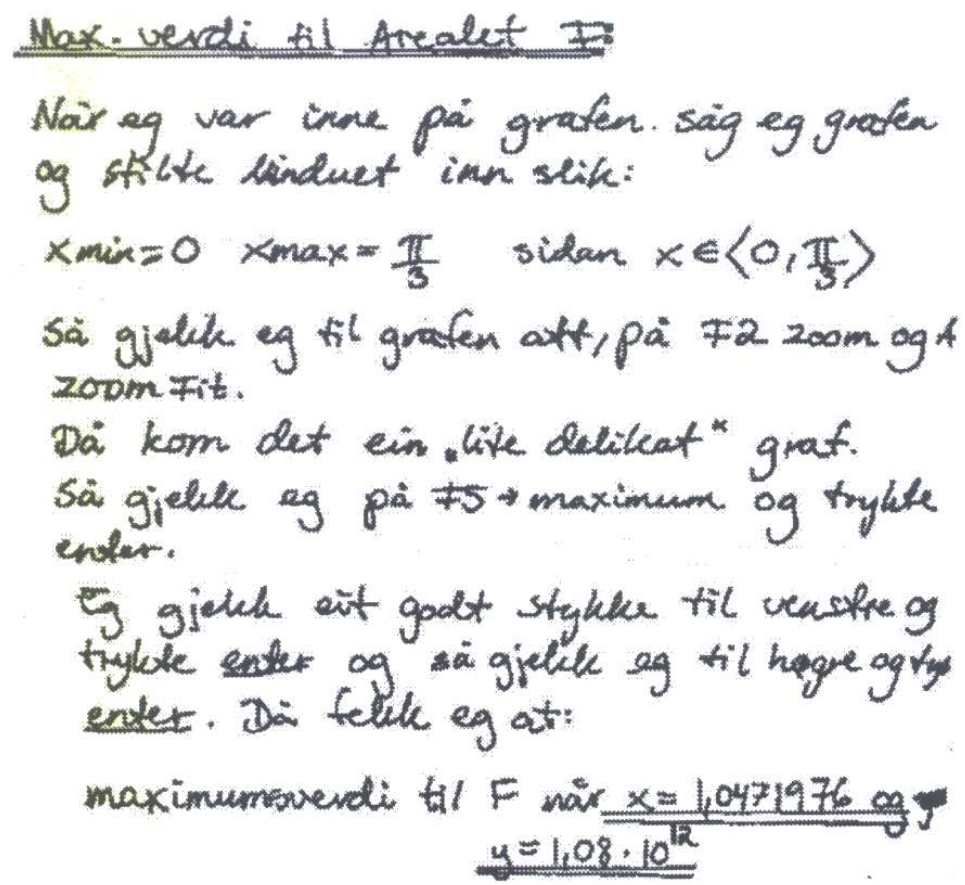

Author's translation:

When I was looking at the graph I adjusted the (view) window as follows: $x \min =0, x \max =\frac{\pi}{3}$ since $x \in\left\langle 0, \frac{\pi}{3}\right\rangle$. Then $I$ went to the graph again, on F2 zoom and A zoom Fit. Then an "indelicate" graph appeared. I 


$$
\text { "gjone" — 2004/7/22 — 15:27 — page } 128 \text { — \#12 }
$$

went to F5 maximum and pressed ENTER. Then I went to the left and pressed ENTER and then to the right and pressed ENTER. Then I got maximum value of $F$ when $x=1,0471976$ and $y=1,08 \cdot 10^{12}$

There is a certain rationale to approach the value $\frac{\pi}{3}$ from left and from right. This would work for finding limits in many cases, and could also be a method when using a calculator to find a limit of an indefinite expression. However, the student should have been suspicious of the answer and tried to use other methods.

The important element for our study is the dependency on the calculator. It was observed for several of the students that they were not trying to reason without the calculator. We might also say that these students were using a procedural approach, and did not attempt a structural approach to the problem.

\section{Discussion}

Let us now return to our questions about procedural and structural knowledge and the relationship to SMC (and graphing calculator). We will consider the use of SMC and graphing calculators for working with functions in the light of the duality between operational and structural knowledge.

Let us start the discussion with the last problem.

The problem in my opinion can be quite easily (as shown above) solved by a "process" - looking for a limit. It is here sufficient to try to insert the value for $x$ in the expression.

The SMC, however, has the capability to compute limits directly, which means that a process approach is not necessary. The students gave the SMC the following expression:

$$
\lim _{x \rightarrow \frac{\pi}{3}} \frac{12.5 \sin (2 x)}{3-4 \sin ^{2} x}
$$

The SMC they used would return "undefined". This might be correct for the limit seen in isolation, but in the actual problem the question to what happened with an area. The answer would be that there is no maximum value for the area. The majority of the students were not able to interpret the result given by the SMC - as an "object" it did not hold a meaning for them.

This illustrates the case that the process aspect would give a more meaningful answer to the problem. The students are trying to work with the limit as an object, using the SMC to find properties of the object. 


$$
\text { "gjone" — 2004/7/22 — 15:27 — page } 129 \text { — \#13 }
$$

Some students, as in the example presented above had a different approach. They were attempting to use a process approach, calculating larger and larger values (areas). Most of them, as in the example failed to observe that there is no maximum value, but was satisfied with a very large number as answer.

It seems that this has some implications for Anna Sfards second principle. Students with the SMC are lead to a structural approach, which is difficult for them to handle.

In the second problem on equality (Problem 4b) the standard solution is a process. We would start out with the expression on the left, and then use transformations to get the other expression.

The SMC has the capability to "investigate" (calculate) limits, giving the answer True or FAlse. Again this leads to a structural approach, as in the discussion above; there is no need of a process approach. When some students have the calculator evaluating the equality it is really closer to considering the equality as an object.

Even if this answer is correct in a sense an important question is also: Is the solution by having the calculator collecting terms "correct"? How is the calculator arriving at the answer? Could we in general trust the test for equality for functions? This leads to the fundamental question how the calculator-algorithm is constructed.

Some students used a graphical approach - drawing both graphs and observing: "they were the same". Looking for differences in the graphs suggests that they considered the graphs as some kind of objects.

This "method" of solving the problem, by observing coinciding graphs on a calculator window, is moreover also a doubtful method. The solution is depending on the resolution of the screen.

It should be noted that the first problem (Problem 1d) ) was a standard type of problem, solved most efficiently with a traditional structural conception of function.

This problem is easily solved by using what I will say is conceptual knowledge about the cosine function (the minimum value is -1 , the maximum value is +1 and the period is $2 \pi$ ). There is however, no standard way to work with the cosine function as an object on the SMC, except for looking at the graph of the function (as an object).

The solution for many of the students, as the one presented above, took the graph (object) as a starting point, and then in a series of steps investigated 


$$
\text { "gjone" — 2004/7/22 — 15:27 — page } 130 \text { — \#14 }
$$

the properties finding MAX/MIN directly or using the TRACE function on the calculator.

Working with functions on a graphing calculator (or SMC) introduces new elements. The graph of a function is automatic from the function expression. Entering

$$
f(x)=8.50+0.70 \cdot \cos \frac{4 \pi}{25} x
$$

gives the graph immediately.

In this example I will argue that the solution based on reading off the graph is based on a procedural knowledge of functions. The students do not see the cosine function as an object with some properties; it is looked upon mainly as a process.

This is also seen in the student's argument. He is eager to present the steps (process), but this is not really a mathematical process. The instructions given in the examination paper asks for the students to explain their steps (solution process) but this student and many others give a very detailed non-mathematical explanation.

So for the problem that has a solution built on structural approach the students tries to use a procedural approach. This will give the correct answer, but is very time consuming.

This might be a case for looking closer at the notion of procept as introduced by Eddie M. Gray and David O. Tall [1]. The symbols in the problems discussed - the limit, the trigonometric equality and the cosine function - all play a dual role representing both a mathematical process to be carried out and the result of that process. Some problems in this test are more efficiently solved with a procedural approach, others more efficiently with a structural approach. To solve such problems efficiently hence requires a flexibility to use different representations.

The introduction of computers in a sense "disturbs" the traditional procedural - structural development. The perspective offered by the notion procept could be more suitable for discussing solving problems by SMCs, where an important element would be the flexibility to combine symbol, process and concept. With SMCs a focus should be proceptual thinking:

Proceptual thinking is characterized by the ability to compress stages in symbol manipulation to the point where symbols are viewed as objects that can be decomposed and recomposed in flexible ways (Gray \& Tall, [1], p. 132). 


$$
\text { "gjone" — 2004/7/22 — 15:27 — page } 131 \text { — \#15 }
$$

The present article gives only isolated examples of students' problem solving. To investigate their thinking further it would be necessary to look at students' solutions on a set of different problems.

\section{Summing up}

The problem we have discussed here is mainly problems about properties of functions.

Investigating and graphing functions the traditional way (on paper) is basically a operational process. Working with functions the structural conception of a function develops gradually through secondary education.

As cited above, Anna Sfard argues that:

Structural conception should not be required as long as the student can

do without it.

In a sense the SMC makes the transition from process to object directly without the student interfering. A function graph on the screen is for many students an object, so is also an equality and a limit.

Many will argue that technology has the possibility of helping students form structural conceptions, but they might be different than the "traditional" ones. In the case of functions perhaps a more graphical structural conception of a function is developed. We can see this aspect clearly in how some calculators handle the graphs of functions. The graphs are objects that can be manipulated. Some graphing calculators also have a kind of "variable graph" function that will vary constants in the equation of the graph.

The question raised in this article is if the introduction of SMCs and also graphing calculators will alter the relationship between procedural and structural knowledge. The transition from a process to an "object" (on the calculator window) is a different kind of development than we have traditionally seen in mathematics education.

\section{References}

[1] E. M. Gray and D. O. Tall, Duality, ambiguity and flexibility: A "proceptual" view of simple arithmetic, Journal for Research in Mathematics Education 25, no. 2 (1994), 116-140.

[2] J. Hiebert and P. Lefevre, Conceptual and procedural knowledge in mathematics: An introductory analysis, Conceptual and procedural knowledge: The case for mathematics, (In J. Hiebert, ed.), Hillsdale, 1986, 1-28, NJ: Lawrence Eribaum. 


$$
\text { "gjone" — 2004/7/22 — 15:27 — page } 132 \text { — \#16 }
$$

[3] M. Niss, Aspects of the nature and state of research in mathematics education, Documenta Mathematica, Extra Volume ICM, (Proceedings of the International Congress of Mathematicians Vol III), 1998, 767-776.

[4] A. Sfard, Transition from operational to structural conception: The notion of function revisited, Proceedings of the Thirteenth International Conference for the Psychology of Mathematics Education, (In G. Vergnaud, J. Rogalski, and M. Artigue, eds.), Paris, France, 1989, 151-158.

[5] A. Sfard, On the dual nature of mathematical conceptions: Reflections on processes and objects as different sides of the same coin, Educational Studies in Mathematics 22 (1991), 1-36.

[6] A. Sfard, On operational-structural duality of mathematical conceptions, Paper presented at PME XVI, New Hampshire (1992).

[7] D. Tall, Information Technology and Mathematics Education: Enthusiasms, Possibilities and Realities, Proceedings of the 8th International Congress on Mathematical Education, (In Alsina, C. (et. al.), eds.), Sevilla: S.A.E.M. 'THALES', 1998.

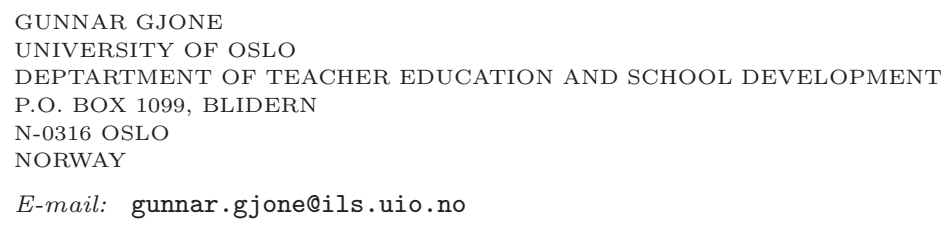

(Received February, 2004) 